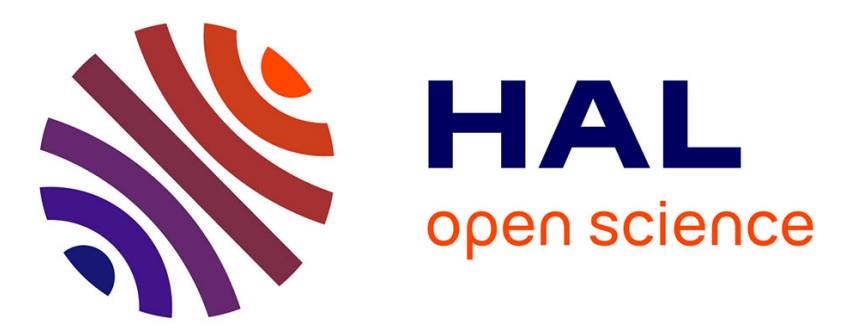

\title{
Robust Fault Detection and Estimation for Descriptor Systems Based on Multi-Models Concept
}

Habib Hamdi, Mickael Rodrigues, Chokri Mechmeche, Naceur Benhadj Braiek

\section{To cite this version:}

Habib Hamdi, Mickael Rodrigues, Chokri Mechmeche, Naceur Benhadj Braiek. Robust Fault Detection and Estimation for Descriptor Systems Based on Multi-Models Concept. International Journal of Control, Automation and Systems, 2012, 10 (6), pp.1260-1266. 10.1007/s12555-012-0622-z . hal00752421

\section{HAL Id: hal-00752421 \\ https://hal.science/hal-00752421}

Submitted on 15 Nov 2012

HAL is a multi-disciplinary open access archive for the deposit and dissemination of scientific research documents, whether they are published or not. The documents may come from teaching and research institutions in France or abroad, or from public or private research centers.
L'archive ouverte pluridisciplinaire HAL, est destinée au dépôt et à la diffusion de documents scientifiques de niveau recherche, publiés ou non, émanant des établissements d'enseignement et de recherche français ou étrangers, des laboratoires publics ou privés. 


\title{
Robust Fault Detection and Estimation for Descriptor Systems based on Multi-Models Concept
}

\author{
H. Hamdi $\dagger$, M. Rodrigues $\ddagger$, C. Mechmeche $\nmid$ and N. Benhadj Braiek $\dagger$
}

\begin{abstract}
This paper addresses the robust fault detection and estimation problem of nonlinear descriptor system with unknown inputs observers. The considered nonlinear descriptor system is transformed into an equivalent multi-models form by using the Takagi-Sugeno (T-S) approach. Two cases are considered: the first one deals with the multi-models with measurable decision variables and the second one assumes that these decision variables are unmeasurable. Then, a residual generator based on an unknown observer is designed for fault detection and estimation. Stability analysis and gain matrices determination are performed by resolving a set of Linear Matrices Inequalities (LMIs) for both cases. The performances of the proposed fault detection and estimation method is successfully applied to an electrical circuit.
\end{abstract}

Keywords: Fault detection and estimation, descriptor multi-models, residual generation, Linear Matrices Inequalities (LMIs).

\section{INTRODUCTION}

It is well recognized that many practical dynamical systems are subject to various environmental changes, unknown disturbances and varying operating conditions. Thus sensors, actuators or components faults in those systems are inevitable. Since any fault in a dynamical system may lead to significant performance degradation, serious system damages and even loss of human life, it is essential to be able to detect and identify faults so that necessary protective actions can be taken in advance. Hence, fault diagnosis of dynamic systems has received more attention and significant progress has been made in recent years for model-based fault diagnosis techniques. More attention has been devoted to the development of robust fault detection methods under external disturbances for continuous time systems modeled by Ordinary Differential Equations (ODE) [15]. Differential-Algebraic Equations (DAEs), singular or descriptor systems are important in fault detection context since DAE systems appear in a large class of technical process like mechanical, electrical and chemical systems $[3,17]$.

With regard to state estimation or controller design, many approaches have been developed to design observers for descriptor systems. In [12], local asymptotic

$\dagger$ H. Hamdi, C. Mechmeche and N. BenHadj Braiek are with Laboratory of Study and Control Automation of Process (LECAP), Polytechnic school of Tunisia (e-mail: hammmdihabib@yahoo.fr,_chokri.mechmeche@esstt.rnu.tn, naceur.benhadj@ept.rnu.tn).

$\ddagger$ M. Rodrigues is with Laboratory Automation and Engineering Process (LAGEP), CNRS UMR 5007,University Lyon1, France (e-mail: mickael.rodrigues@univ-lyon1.fr). observer is obtained for general nonlinear descriptor systems by means of a coordinate transformation. [6] studies observer issued for continuous nonlinear descriptor systems via a convex optimization. In addition in [10], a robust extended strictly positive real (ESPR) analysis and control for continuous-time descriptor systems with norm-bounded uncertainty was studied.

Until now, the design of fault diagnosis algorithm is a crucial problem for several physical process that are modeled by nonlinear DAEs [21]. The expansion of faults diagnosis approaches of ordinary systems $[14,15]$ to descriptor systems, requires to resolve DAEs on line. In practice, it may not be possible to employ standard estimation schemes described by DAEs for on line health monitoring due to difficulties in solving DAEs. Therefore, it is highly desirable to develop fault diagnosis algorithms that are based on ordinary differential equations. The design of these algorithms can become very difficult even impossible according to the type and the complexity of the employed model, from where the importance to have a mathematical model of the system where it's at the same time, simple and precise. The multi-models approach is a powerful technique of modeling nonlinear systems which make it possible to get a good compromise between the precision and the complexity of the model [14]. Multi-models are recognized for their capacity to take into account the changes in the operating mode of the system and to reproduce its behavior with precision in a broad operating range. Moreover, they offer mathematical properties which can be profitable during the design of observers. More recently, a lot of works have appeared that consider fault detection and estimation problem for multi-models 
systems [7]. Nevertheless, the authors in [5] have developed an observer for robust residual generation which minimizes the sensitivity to the disturbances and maximizes the sensitivity to the faults. In [16] the authors have synthesized observers for nonlinear systems modeled by decoupled multi-models. However, all these previous results have been realized only for regular systems and not for descriptor systems.

For descriptor systems, a few works on fuzzy control have attracted a lot of attention during the last few years $[18,19]$. Concerning the fault diagnosis problems, very few results have been generalized to the descriptor multi-models. In [8] and [9] the problem of fault detection isolation and estimation for LPV polytopic descriptor system has been studied by using unknown inputs observer and proportional integral observer respectively.

In fact several works are devoted to the state estimation by the use of multi-models with measurable decision variables which are especially represented by the input variables or the outputs of the system. Unfortunately, in many situations, these decision variables are the state variables, whose measurements are not always available. Only few results like [5] are dedicated to the use of these models for state estimation and in [4] for fault estimation. But, these results were only obtained for ordinary nonlinear systems and from the authors' knowledge and besides the recent new paper by [2] which offers an unknown input observer for descriptor multi-models with unmeasurable decision variables, no work addresses the state estimation and fault estimation for singular multi-models with unmeasurable decision variables.

So, this paper presents some novelty by the study of singular systems represented by descriptor multi-models with unmeasurable decision variables which generalize the well-known multi-models approach for ordinary systems. Moreover, this paper brings some new results for the state estimation and fault estimation dedicated to descriptor multi-models with unmeasurable decision variables. Then, in a first step, an unknown input observer is designed in order to detect actuator faults based on descriptor multi-models subject to disturbances. In a second step, an augmented observer for an augmented singular multi-models with unmeasurable decision variables is proposed for fault estimation. The presented fault estimation concept is a generalized study of a particular class of nonlinear descriptor system under Lipschitz constraints proposed by [20,21] when the results are valid only in a neighborhood of a single nominal operating point. However, this concept has not been adapted for regular multi-models synthesis either for descriptor multi-models with measured or unmeasured decision variable. So, this paper aims at developing this strategy for such systems. Moreover, the stability analysis of the dynamic state estimation error is performed by using the Lyapunov method for two cases; the first one concerns the descriptor multi-models with measurable decision variable and the second case is made with unmeasurable decision variable. These two cases have been realized through the use of a set of Linear Matrices Inequalities (LMIs).

The paper is organized as follows: the structure of the studied systems is defined in section 2 and the design method to detect faults and stability analysis are detailed in section 3. Section 4 is dedicated to reconstruct the fault by using an augmented form of the faulty system and a dedicated unknown inputs observer is designed. Before ending with some conclusions, the proposed singular multi-models is applied to an electrical circuit and observers are designed in section 5 in order to illustrate the effectiveness of the proposed method.

In this paper, the notation is standard. $\mathbb{R}$ denotes the set of real numbers, $\mathscr{C}$ is the complex plane, $A^{+}$denotes the generalized inverse of $A ; Q>0$ or $(Q<0)$ indicates the symmetric matrix $Q$ is positive (or negative) definite; $\|$.$\| denotes the standard norm symbol. Also, \forall$ means "for all".

\section{DESCRIPTOR MULTI-MODELS STRUCTURE}

Consider the following continuous-time nonlinear descriptor system:

$$
\left\{\begin{array}{l}
E \dot{x}(t)=\mathscr{F}(x(t), u(t), d(t)) \\
y(t)=\mathscr{G}(x(t), u(t), d(t))
\end{array}\right.
$$

where $x(t) \in \mathbb{R}^{n}$ is the singular state vector, $u(t) \in \mathbb{R}^{p}$ is the input vector, $y(t) \in \mathbb{R}^{m}$ is the output vector. $\mathscr{F}($.$) and$ $\mathscr{G}($.$) are continuous and infinitely differentiable nonlin-$ ear functions. $\mathrm{E}$ is a singular matrix with constant parameters and $\operatorname{rank}(E)=r$.

The multi-models approach allows to represent nonlinear systems into a convex combination of linear submodels $[2,15]$. Let us consider the nonlinear descriptor system (1) represented by a multi-models as follows:

$$
\left\{\begin{array}{c}
E \dot{x}(t)=\sum_{i=1}^{h} h_{i}(\xi(t))\left[A_{i} x(t)+B_{i} u(t)+R_{i} d(t)\right. \\
y(t)=C x(t)+G d(t)
\end{array}\right.
$$

where $A_{i}, B_{i}, R_{i}, F_{i}, C$ and $G$ are jacobian matrices relating to the $i^{\text {th }}$ operating point. $\Delta x_{i}$ is a vector witch depends on the $i^{t h}$ operating point. $d(t) \in \mathbb{R}^{q}(q \leq m)$ is a disturbance vector and $f(t) \in \mathbb{R}^{k}$ is a fault vector. $\xi(t)$ is a decision variable (also called premise variable [2]) that can be measurable or unmeasurable. $h_{i}(\xi(t))$ are the weighting functions that measure the relative contribution of each local model to build the global model. The 
functions $h_{i}(\xi(t))$ check the properties of the following convex set:

$$
\left\{\begin{array}{l}
\sum_{i=1}^{h} h_{i}(\xi(t))=1 \\
0 \leq h_{i}(\xi(t)) \leq 1
\end{array}\right.
$$

The multi-models structure makes possible to provide a mean of generalization of tools developed for linear systems to nonlinear systems. The theory for the observer design is derived for singular systems under the assumption that the system has the global observability. This concept implies the observability of the algebraic part and the differential part of a descriptor system $[2,13]$. Then, the observability for descriptor multi-models notion requires that all the subsystems are observable [8]. Assumptions $A 2$ and $A 3$ extend the observability properties to the descriptor multi-models.

Assumption A1: $\operatorname{rank}\left(C R_{i}\right)=\operatorname{rank}\left(R_{i}\right)$.

Assumption A2: The row vectors of the matrices $C$ and $E$ must be a basis of the n-dimensional vector space:

$$
\operatorname{rank}\left[\begin{array}{c}
E \\
C
\end{array}\right]=n
$$

Assumption A3: Each local model of the descriptor multi-models (2), has to be observable $\forall i=1, \ldots, h$.

$$
\operatorname{rank}\left[\begin{array}{c}
s E-A_{i} \\
C
\end{array}\right]=n, \forall s \in \mathscr{C} \text {. }
$$

The following section is dedicated to generate an alarm signal when a fault occurs and produce an accurate estimate of the fault vector $f(t)$.

\section{ROBUST FAULT DETECTION IN DESCRIPTOR MULTI-MODELS}

In this section, the design of a residual generator based on unknown input observer for descriptor multimodels is developped. The global residual generator is defined by:

$$
\left\{\begin{array}{l}
\dot{z}(t)=\sum_{i=1}^{h} h_{i}(\xi(t))\left[N_{i} z(t)+G_{i} u(t)+L_{i} y(t)+\Delta z_{i}\right] \\
\hat{x}(t)=z(t)+T_{2} y(t) \\
r(t)=M_{1} z(t)+M_{2} y(t)
\end{array}\right.
$$

where $r(t)$ is the residual vector, $z(t)$ and $\hat{x}(t)$ are the state vector and the estimated state vector respectively. The residual generator design is reduced to determine the gains $N_{i}, G_{i}, \Delta z_{i}, L_{i}, M_{1}, T_{2}$ and $M_{2}$ such that the state estimation error is stable. Then, to analyze the convergence of the residual generator, let consider the estimation error from (2) and (6) such that:

$$
\begin{gathered}
e(t)=\hat{x}(t)-x(t) \\
e(t)=z(t)-\left(I_{n}-T_{2} C\right) x(t)+T_{2} G d(t)
\end{gathered}
$$

Under Assumption $A 2$, there exists nonsingular matrices $T_{1} \in \mathbb{R}^{n \times n}$ and $T_{2} \in \mathbb{R}^{n \times m}$ such that:

$$
T_{1} E+T_{2} C=I_{n}
$$

The dynamic estimation error is then described by:

$$
\dot{e}(t)=\dot{z}(t)-T_{1} E \dot{x}(t)+T_{2} G \dot{d}(t)
$$

which is equivalent to

$$
\begin{aligned}
& \dot{e}(t)=\sum_{i=1}^{h} h_{i}(\xi(t))\left[N_{i} z(t)+G_{i} u(t)+L_{i} y(t)+\Delta z_{i}\right. \\
& \left.-T_{1} A_{i} x(t)-T_{1} B_{i} u(t)-T_{1} R_{i} d(t)-T_{1} F_{i} f(t)-T_{1} \Delta x_{i}\right]
\end{aligned}
$$

The estimation error is finally given by:

$$
\begin{aligned}
& \dot{e}(t)=\sum_{i=1}^{h} h_{i}(\xi(t))\left[N_{i} e(t)+\left(N_{i} T_{1} E+L_{i} C-T_{1} A_{i}\right) x(t)\right. \\
& +\left(G_{i}-T_{1} B_{i}\right) u(t)+\left(L_{i} G-N_{i} T_{2} G-T_{1} R_{i}\right) d(t) \\
& \left.-T_{1} F_{i} f(t)+T_{2} G \dot{d}(t)+\Delta z_{i}-T_{1} \Delta x_{i}\right]
\end{aligned}
$$

From (6), the general expression of the residual vector can be written as:

$$
r(t)=M_{1} z(t)+M_{2} y(t)
$$

and by using the estimation error (7), it follows:

$$
\begin{array}{r}
r(t)=M_{1} e(t)+\left(M_{1} T_{1} E+M_{2} C\right) x(t) \\
+\left(M_{2}-M_{1}\right) T_{2} G d(t)
\end{array}
$$

If the following conditions hold true $\forall i=1, \ldots, h$ :

$$
\begin{aligned}
& N_{i} T_{1} E+L_{i} C-T_{1} A_{i}=0 \\
& M_{1} T_{1} E+M_{2} C=0 \\
& G_{i}-T_{1} B_{i}=0 \\
& L_{i} G-N_{i} T_{2} G-T_{1} R_{i}=0 \\
& T_{2} G=0 \\
& \Delta z_{i}-T_{1} \Delta x_{i}=0
\end{aligned}
$$

then, the estimated error $e(t)$ and residual $r(t)$ become:

$$
\begin{aligned}
\dot{e}(t) & =\sum_{i=1}^{h} h_{i}(\xi(t))\left[N_{i} e(t)-T_{1} F_{i} f(t)\right] \\
r(t) & =M_{1} e(t)
\end{aligned}
$$

Therefore, the design of the residual generator (6) is reduced to satisfy the constraints (14) to (19) by taking into the stability of (20). In order to establish the gains matrices of the designed residual generator, the substitution of (8) into (14) yields to:

$$
\begin{aligned}
& T_{1} A_{i}=N_{i}\left(I_{n}-T_{2} C\right)+L_{i} C \\
& N_{i}=T_{1} A_{i}+\left(N_{i} T_{2}-L_{i}\right) C \\
& N_{i}=T_{1} A_{i}+K_{i} C
\end{aligned}
$$

where

$$
K_{i}=N_{i} T_{2}-L_{i}
$$


To design matrices $T_{1}$ and $T_{2}$, let consider equation (8) under equality (18), one can write the following relationship:

$$
\left[\begin{array}{ll}
T_{1} & T_{2}
\end{array}\right]\left[\begin{array}{ll}
E & 0 \\
C & G
\end{array}\right]=\left[\begin{array}{ll}
I_{n} & 0
\end{array}\right]
$$

Under Assumption $A 1$, a solution $\left[\begin{array}{cc}T_{1} & T_{2}\end{array}\right]$ exists if and only if the matrix $\left[\begin{array}{cc}E & 0 \\ C & G\end{array}\right]$ is a full column rank [13]. Then, a particular solution of (25) is given by using the generalized inverse matrix such that:

$$
\left[\begin{array}{ll}
T_{1} & T_{2}
\end{array}\right]=\left[\begin{array}{ll}
I_{n} & 0
\end{array}\right]\left[\begin{array}{ll}
E & 0 \\
C & G
\end{array}\right]^{+}
$$

then, by takeing,

$$
T=\left[\begin{array}{ll}
I_{n} & 0
\end{array}\right]\left[\begin{array}{ll}
E & 0 \\
C & G
\end{array}\right]^{+} \in \mathbb{R}^{n \times(n+m)}
$$

we can write:

$$
T=\left[\begin{array}{cc}
1, \ldots, n & n+1, \ldots, n+m \\
T_{1} & T_{2}
\end{array}\right]
$$

After, to compute matrices $M_{1}$ and $M_{2}$, equation (15) can be rewritten as:

$$
\left[\begin{array}{ll}
M_{1} & M_{2}
\end{array}\right]\left[\begin{array}{c}
T_{1} E \\
C
\end{array}\right]=0
$$

To solve equation (28), it is equivalent to write:

$$
\left[\begin{array}{c}
T_{1} E \\
C
\end{array}\right]^{T}\left[\begin{array}{ll}
M_{1}^{T} & M_{2}^{T}
\end{array}\right]^{T}=0
$$

Hence, (29) admits a solution $\left[\begin{array}{ll}M_{1}^{T} & M_{2}^{T}\end{array}\right]^{T}$ in the null space of $\left[\begin{array}{c}T_{1} E \\ C\end{array}\right]^{T}$ i.e.:

$$
\left[\begin{array}{ll}
M_{1}^{T} & M_{2}^{T}
\end{array}\right]^{T} \subseteq \operatorname{Ker}\left[\begin{array}{c}
T_{1} E \\
C
\end{array}\right]^{T}
$$

Then, a solution can be chosen as follows:

$$
\left[\begin{array}{ll}
M_{1}^{T} & M_{2}^{T}
\end{array}\right]^{T}=\operatorname{ker}\left[\begin{array}{c}
T_{1} E \\
C
\end{array}\right]^{T}
$$

if we express

$$
\Delta^{T}=\operatorname{ker}\left[\begin{array}{c}
T_{1} E \\
C
\end{array}\right]^{T}
$$

it's equivalently to,

$$
\Delta^{T}=\left[\begin{array}{ll}
M_{1}^{T} & M_{2}^{T}
\end{array}\right]^{T}
$$

then, one can write:

$$
\Delta=\left[\begin{array}{cc}
1, \ldots, n & n+1, \ldots, n+m \\
M_{1} & M_{2}
\end{array}\right]
$$

\subsection{Design and stability analysis}

In this subsection, the stability problem is studied for two cases:

Case A: when the decision variable of the weighting function depends of a measured state variable.

Case B: when this variable depends of an unmeasured state variable.

\section{Case A: Measurable decision variable}

From the following dynamic state estimation error:

$$
\dot{e}(t)=\sum_{i=1}^{h} h_{i}(\xi(t))\left[N_{i} e(t)-T_{1} F_{i} f(t)\right]
$$

and by using (24), it becomes:

$$
\dot{e}(t)=\sum_{i=1}^{h} h_{i}(\xi(t))\left[\left(T_{1} A_{i}+K_{i} C\right) e(t)-T_{1} F_{i} f(t)\right]
$$

The stability of the dynamic estimation error (34) can be verified by the following Theorem.

Theorem 1: The estimation error equation (34) is globally asymptotically stable, if the fault $f(t)$ satisfies $\|f(t)\| \leq \mu, \mu>0$ and if there exists real parameters $\alpha$ and $\beta$, a common positive definite matrix $Q$ and matrices $W_{i}=Q K_{i}$ such that, $\forall i=1, \ldots, h$ :

$$
\left[\begin{array}{cc}
\left(T_{1} A_{i}\right)^{T} Q+Q\left(T_{1} A_{i}\right)+\alpha I_{n}+C^{T} W_{i}^{T}+W_{i} C & Q\left(T_{1} F_{i}\right) \\
\left(T_{1} F_{i}\right)^{T} Q & -\frac{1}{\beta} I_{k}
\end{array}\right]<0
$$

s.t.

$$
W_{i} G+Q T_{1} R_{i}=0
$$

\section{Proof:}

Consider the Lyapunov function with the following quadratic form:

$$
V(e(t))=e^{T}(t) Q e(t)>0
$$

Stability condition for the estimation error yields to that the time derivative of the Lyapunov function should be negative definite over (3). The derivative of $V(e(t))$ with respect to time evaluated on the trajectories of the error equation (33) is:

$$
\begin{aligned}
\dot{\mathrm{V}}(\mathrm{e})=\sum_{\mathrm{i}=1}^{\mathrm{h}} \mathrm{h}_{\mathrm{i}}(\xi(\mathrm{t})) & \left\{\mathrm{e}^{\mathrm{T}}(\mathrm{t})\left[\mathrm{N}_{\mathrm{i}}^{\mathrm{T}} \mathrm{Q}+\mathrm{QN}_{\mathrm{i}}\right] \mathrm{e}(\mathrm{t})\right. \\
& \left.-2 \mathrm{e}^{\mathrm{T}}(\mathrm{t}) \mathrm{Q}\left(\mathrm{T}_{1} \mathrm{~F}_{\mathrm{i}}\right) \mathrm{f}(\mathrm{t})\right\}
\end{aligned}
$$

If $\|f(t)\| \leq \mu$, then

$$
\begin{array}{r}
\dot{\mathrm{V}}(\mathrm{e}(\mathrm{t})) \leq \sum_{\mathrm{i}=1}^{\mathrm{h}} \mathrm{h}_{\mathrm{i}}(\xi(\mathrm{t}))\left\{\mathrm{e}^{\mathrm{T}}(\mathrm{t})\left[\mathrm{N}_{\mathrm{i}}^{\mathrm{T}} \mathrm{Q}+\mathrm{QN}_{\mathrm{i}}\right] \mathrm{e}(\mathrm{t})\right. \\
\left.-2 \mu\left\|\mathrm{e}^{\mathrm{T}}(\mathrm{t}) \mathrm{Q}\left(\mathrm{T}_{1} \mathrm{~F}_{\mathrm{i}}\right)\right\|\right\}
\end{array}
$$


Since $\mu>0$, the derivative of $V(e(t))$ becomes:

$$
\begin{array}{r}
\dot{\mathrm{V}}(\mathrm{e}(\mathrm{t}))<\sum_{\mathrm{i}=1}^{\mathrm{h}} \mathrm{h}_{\mathrm{i}}(\xi(\mathrm{t}))\left\{\mathrm{e}^{\mathrm{T}}(\mathrm{t})\left[\mathrm{N}_{\mathrm{i}}^{\mathrm{T}} \mathrm{Q}+\mathrm{QN}_{\mathrm{i}}\right] \mathrm{e}(\mathrm{t})\right. \\
\left.+2 \mu\left\|\mathrm{e}^{\mathrm{T}}(\mathrm{t}) \mathrm{Q}\left(\mathrm{T}_{1} \mathrm{~F}_{\mathrm{i}}\right)\right\|\right\}
\end{array}
$$

For any positive scalar $\alpha$, we have the following inequality:

$$
2 \mu\left\|\mathrm{e}^{\mathrm{T}}(\mathrm{t}) \mathrm{Q}\left(\mathrm{T}_{1} \mathrm{~F}_{\mathrm{i}}\right)\right\| \leq \alpha^{-1} \mu^{2}\left\|\mathrm{e}^{\mathrm{T}}(\mathrm{t}) \mathrm{Q}\left(\mathrm{T}_{1} \mathrm{~F}_{\mathrm{i}}\right)\right\|^{2}+\alpha
$$

Hence,

$$
\begin{aligned}
& \dot{\mathrm{V}}(\mathrm{e}(\mathrm{t}))<\sum_{\mathrm{i}=1}^{\mathrm{h}} \mathrm{h}_{\mathrm{i}}(\xi(\mathrm{t}))\left\{\mathrm{e}^{\mathrm{T}}(\mathrm{t})\left[\mathrm{N}_{\mathrm{i}}^{\mathrm{T}} \mathrm{Q}+\mathrm{QN}_{\mathrm{i}}\right] \mathrm{e}(\mathrm{t})\right. \\
&\left.+\alpha^{-1} \mu^{2}\left\|\mathrm{e}^{\mathrm{T}}(\mathrm{t}) \mathrm{Q}\left(\mathrm{T}_{1} \mathrm{~F}_{\mathrm{i}}\right)\right\|^{2}+\alpha\right\}
\end{aligned}
$$

Let take $\beta=\alpha^{-1} \mu^{2}$ then:

$$
\begin{aligned}
\dot{\mathrm{V}}(\mathrm{e}(\mathrm{t}))< & \sum_{\mathrm{i}=1}^{\mathrm{h}} \mathrm{h}_{\mathrm{i}}(\xi(\mathrm{t}))\left\{\mathrm{e}^{\mathrm{T}}(\mathrm{t})\left[\mathrm{N}_{\mathrm{i}}^{\mathrm{T}} \mathrm{Q}+\mathrm{QN}\right] \mathrm{e}(\mathrm{t})\right. \\
& \left.+\beta \mathrm{e}^{\mathrm{T}}(\mathrm{t}) \mathrm{Q}\left(\mathrm{T}_{1} \mathrm{~F}_{\mathrm{i}}\right)\left(\mathrm{T}_{1} \mathrm{~F}_{\mathrm{i}}\right)^{\mathrm{T}} \mathrm{Qe}(\mathrm{t})+\alpha\right\} \\
\dot{\mathrm{V}}(\mathrm{e}(\mathrm{t}))< & \sum_{\mathrm{i}=1}^{\mathrm{h}} \mathrm{h}_{\mathrm{i}}(\xi(\mathrm{t}))\left\{\mathrm { e } ^ { \mathrm { T } } ( \mathrm { t } ) \left[\mathrm{N}_{\mathrm{i}}^{\mathrm{T}} \mathrm{Q}+\mathrm{QN}_{\mathrm{i}}\right.\right. \\
& \left.\left.+\beta \mathrm{Q}\left(\mathrm{T}_{1} \mathrm{~F}_{\mathrm{i}}\right)\left(\mathrm{T}_{1} \mathrm{~F}_{\mathrm{i}}\right)^{\mathrm{T}} \mathrm{Q}\right] \mathrm{e}(\mathrm{t})+\alpha\right\}
\end{aligned}
$$

$\forall i=1, \ldots, h \sum_{i=1}^{h} h_{i}(\xi(t))=1$ and $h_{i}(\xi(t)) \geq 0$, the inequality (38) is definite negative if:

$$
N_{i}^{T} Q+Q N_{i}+\beta Q\left(T_{1} F_{i}\right)\left(T_{1} F_{i}\right)^{T} Q+\alpha I_{n}<0
$$

While replacing $N_{i}$ by the expression (24) and for $W_{i}=$ $Q K_{i}$, the last inequality can be written $\forall i=1, \ldots, h$ such that:

$$
\left(T_{1} A_{i}\right)^{T} Q+Q\left(T_{1} A_{i}\right)+\alpha I_{n}+\left(W_{i} C\right)^{T}+W_{i} C+\beta Q\left(T_{1} F_{i}\right)\left(T_{1} F_{i}\right)^{T} Q<0
$$

Using Schur complement, the last inequality becomes $\forall i=1, \ldots, h$ :

$$
\left[\begin{array}{cc}
\left(T_{1} A_{i}\right)^{T} Q+Q\left(T_{1} A_{i}\right)+\alpha I_{n}+C^{T} W_{i}^{T}+W_{i} C & Q\left(T_{1} F_{i}\right) \\
\left(T_{1} F_{i}\right)^{T} Q & -\frac{1}{\beta} I_{k}
\end{array}\right]<0
$$

The obtained gains matrices $K_{i}$ must satisfied the constraint (17) $\forall i=1, \ldots, h$. Then, the equation (17) can be written as follows:

$$
\left(N_{i} T_{2}-L_{i}\right) G+T_{1} R_{i}=0
$$

and under equation (24), it can be expressed as:

$$
K_{i} G+T_{1} R_{i}=0
$$

for $K_{i}=Q^{-1} W_{i} \forall i=1, \ldots, h$, equation (42) becomes:

$$
W_{i} G+Q T_{1} R_{i}=0
$$

Then, gains matrices $K_{i}$ will be obtained by solving LMIs (40) under constraint (43). Therefore, Theorem 1 implies that the state estimation error $e(t)$ is globally asymptotically stable in spite of the presence of fault which acts like additive input.
Case B: Unmeasurable decision variable

For Case B, the descriptor multi-models (2) can be rewritten [5] as:

$$
\left\{\begin{array}{c}
E \dot{x}(t)=\sum_{i=1}^{h} h_{i}(\hat{x}(t))\left[A_{i} x(t)+B_{i} u(t)+R_{i} d(t)\right. \\
y(t)=C x(t)+G d(t)
\end{array}\right.
$$

where

$$
\begin{gathered}
w(t)=\sum_{i=1}^{h}\left(h_{i}(x(t))-h_{i}(\hat{x}(t))\right)\left[A_{i} x(t)+B_{i} u(t)\right. \\
\left.+R_{i} d(t)+F_{i} f(t)+\Delta x_{i}\right]
\end{gathered}
$$

and $w(t)$ acts like a disturbance on the dynamic of the descriptor multi-models. The dynamic of the estimation error becomes:

$$
\dot{e}(t)=\sum_{i=1}^{h} h_{i}(\hat{x}(t))\left(N_{i} e(t)-T_{1} F_{i} f(t)-T_{1} w(t)\right)
$$

Then, to ensure stability of the estimation error (46) when $w(t)=0$ and to attenuate the influence of the disturbance when $w(t) \neq 0$, the $\mathrm{L}_{2}$ approach is used.

Theorem 2: The estimation error (46) is globally asymptotically stable if the term $w(t)$ and $f(t)$ satisfies respectively $\|e(t)\|_{2} \leq \gamma\|w(t)\|_{2}$ and $\|f(t)\| \leq \mu$ and if the conditions (14) to (19) hold and if there exists real parameters $\alpha$ and $\beta$, a common positive definite matrix $Q$ and matrices $W_{i}=Q K_{i}$ such that $\forall i=1, \ldots, h$ :

$$
\left[\begin{array}{ccc}
\Pi_{i} & Q\left(T_{1} F_{i}\right) & -Q T_{1} \\
\left(T_{1} F_{i}\right)^{T} Q & -\frac{1}{\beta} I_{k} & 0 \\
-T_{1}^{T} Q & 0 & -\delta I_{n}
\end{array}\right]<0
$$

$$
W_{i} G+Q T_{1} R_{i}=0
$$

where

$\Pi_{i}=\left(T_{1} A_{i}\right)^{T} Q+Q\left(T_{1} A_{i}\right)+\alpha I_{n}+\left(W_{i} C\right)^{T}+\left(W_{i} C\right)+I_{n}$ and $\delta=\gamma^{2}$

\section{Proof:}

To find convergence conditions, a Lyapunov quadratic function is chosen as: $V(e)=e^{T}(t) Q e(t)$, differentiating it along (46) and using (38), it becomes:

$$
\begin{aligned}
\dot{\mathrm{V}}(\mathrm{e}(\mathrm{t})) \leq & \sum_{\mathrm{i}=1}^{\mathrm{h}} \mathrm{h}_{\mathrm{i}}(\xi(\mathrm{t}))\left\{\mathrm{e}^{\mathrm{T}}(\mathrm{t})\left[\mathrm{N}_{\mathrm{i}}^{\mathrm{T}} \mathrm{Q}+\mathrm{QN} \mathrm{N}_{\mathrm{i}}\right] \mathrm{e}(\mathrm{t})\right. \\
& +\beta \mathrm{e}^{\mathrm{T}}(\mathrm{t}) \mathrm{Q}\left(\mathrm{T}_{1} \mathrm{~F}_{\mathrm{i}}\right)\left(\mathrm{T}_{1} \mathrm{~F}_{\mathrm{i}}\right)^{\mathrm{T}} \mathrm{Qe}(\mathrm{t})+\alpha \\
& \left.-\mathrm{e}^{\mathrm{T}}(\mathrm{t}) \mathrm{QT}_{1} \mathrm{w}(\mathrm{t})-\mathrm{w}^{\mathrm{T}}(\mathrm{t}) \mathrm{T}_{1}^{\mathrm{T}} \mathrm{Qe}(\mathrm{t})\right\}
\end{aligned}
$$

In order to attenuate the effect of $w(t)$ on the state estimation error, we use a $\mathrm{L}_{2}$ approach. The aim is to minimize the $\mathrm{L}_{2}$ gain from $w(t)$ to $e(t)$ is bounded by $\gamma$ i.e: $\|e(t)\|_{2} \leq \gamma\|w(t)\|_{2}, \gamma>0$. Then, the $\mathrm{L}_{2}$ gain from $e(t)$ to $w(t)$ is bounded by $\gamma$ if:

$$
\dot{V}(e(t))-\gamma^{2} w^{T}(t) w(t)+e^{T}(t) e(t)<0
$$


By substituting $\dot{V}(e(t))$, we obtain:

$$
\begin{aligned}
\sum_{\mathrm{i}=1}^{\mathrm{h}} \mathrm{h}_{\mathrm{i}}(\xi(\mathrm{t}))\left\{\mathrm{e}^{\mathrm{T}}(\mathrm{t})\left[\mathrm{N}_{\mathrm{i}}^{\mathrm{T}} \mathrm{Q}+\mathrm{QN}_{\mathrm{i}}\right] \mathrm{e}(\mathrm{t})\right. \\
+\beta \mathrm{e}^{\mathrm{T}}(\mathrm{t}) \mathrm{Q}\left(\mathrm{T}_{1} \mathrm{~F}_{\mathrm{i}}\right)\left(\mathrm{T}_{1} \mathrm{~F}_{\mathrm{i}}\right)^{\mathrm{T}} \mathrm{Qe}(\mathrm{t})+\alpha \\
-\mathrm{e}^{\mathrm{T}}(\mathrm{t}) \mathrm{QT} \mathrm{T}_{1} \mathrm{w}(\mathrm{t})-\mathrm{w}^{\mathrm{T}}(\mathrm{t}) \mathrm{T}_{1}^{\mathrm{T}} \mathrm{Qe}(\mathrm{t}) \\
\left.\quad-\gamma^{2} \mathrm{w}^{\mathrm{T}}(\mathrm{t}) \mathrm{w}(\mathrm{t})+\mathrm{e}^{\mathrm{T}}(\mathrm{t}) \mathrm{e}(\mathrm{t})\right\}<0
\end{aligned}
$$

The previous inequality can be written as follows $\forall i=$ $1, \ldots, h$ :

$$
\Omega^{\mathrm{T}} \Psi_{i} \Omega<0
$$

where

$$
\begin{gathered}
\Omega^{\mathrm{T}}=\left[\begin{array}{cc}
e^{T}(t) & w^{T}(t)
\end{array}\right] \\
\Psi_{i}=\left[\begin{array}{cc}
N_{i}^{T} Q+Q N_{i}+\alpha I_{n}+\beta Q\left(T_{1} F_{i}\right)\left(T_{1} F_{i}\right)^{T} Q+I_{n} & -Q T_{1} \\
-T_{1}^{T} Q & -\gamma^{2} I_{n}
\end{array}\right] \\
\text { and } \quad \Omega=\left[\begin{array}{c}
e(t) \\
w(t)
\end{array}\right]<0
\end{gathered}
$$

So, $\dot{V}(e(t))<0$ if, $\forall i=1, \ldots, h$ :

$$
\left[\begin{array}{cc}
N_{i}^{T} Q+Q N_{i}+\alpha I_{n}+\beta Q\left(T_{1} F\right)\left(T_{1} F_{i}\right)^{T} Q+I_{n} & -Q T_{1} \\
-T_{1}^{T} Q & -\gamma^{2} I_{n}
\end{array}\right]<0
$$

To obtain an equivalent constraint LMI, one can pose the change of variables as: $W_{i}=Q K_{i}$ and by using the Schur complement, the inequality (52) becomes

$$
\left[\begin{array}{ccc}
\Pi_{i} & Q\left(T_{1} F_{i}\right) & -Q T_{1} \\
\left(T_{1} F_{i}\right)^{T} Q & -\frac{1}{\beta} I_{k} & 0 \\
-T_{1}^{T} Q & 0 & -\delta I_{n}
\end{array}\right]<0
$$

where

$\Pi_{i}=\left(T_{1} A_{i}\right)^{T} Q+Q\left(T_{1} A_{i}\right)+\alpha I_{n}+\left(W_{i} C\right)^{T}+\left(W_{i} C\right)+I_{n}$ and $\delta=\gamma^{2}$.

Now, as shown in the proof of Theorem (1), the obtained matrices $K_{i}$ must satisfy the constraints (17) or its equivalent (43). For this way, the linear inequality (53) can be solved in $Q$ and $W_{i}$ via numerical approach within the LMI framework under constraint (43). The existence of those resulted matrices ensure the stability of the estimation error.

\section{ROBUST FAULT ESTIMATION}

To estimate the fault after the alarm has been generated, we consider the following descriptor multi-models subjects to faults $f(t)$ and disturbances $d(t)$ given by:

$$
\left\{\begin{array}{c}
E \dot{x}(t)=\sum_{i=1}^{h} h_{i}(\hat{x}(t))\left[A_{i} x(t)+B_{i} u(t)+R_{i} d(t)\right. \\
\left.y(t)=C x(t)+G d(t) \quad+F_{i} f(t)+\Delta x_{i}+w(t)\right]
\end{array}\right.
$$

where $h_{i}(\hat{x}(t))$ is a weighting function depend on the unmeasurable state. Let us assume that $f(t)$ is differentiable and:

$$
\zeta_{j}(t)=f^{(l-j)}(t)
$$

where $l$ is the class of $f(t)$ and $j=1, \ldots, l$ is the $j^{t h}$ derivative of this fault.The following relationships [20] hold:

$$
\left\{\begin{array}{l}
\dot{\zeta}_{1}(t)=f^{(l)}(t) \\
\dot{\zeta}_{2}(t)=f^{(l-1)}(t)=\zeta_{1}(t) \\
\vdots \\
\dot{\zeta}_{l}(t)=f(t)=\zeta_{l-1}(t)
\end{array}\right.
$$

The descriptor multi-models (54) and the equations set (56) can be written in an augmented form as follows:

$$
\left\{\begin{array}{l}
\bar{E} \dot{\bar{x}}(t)=\sum_{i=1}^{h} h_{i}(\hat{x}(t))\left[\bar{A}_{i} \bar{x}(t)+\bar{B}_{i} u(t)+\bar{R}_{i} \bar{d}(t)\right. \\
y(t)=\bar{C} \bar{x}(t)=\bar{G} \bar{d}(t)
\end{array}\right.
$$

where

$$
\bar{x}^{T}(t)=\left[\begin{array}{llll}
x^{T}(t) & \zeta_{1}^{T}(t) & \ldots & \zeta_{l}^{T}(t)
\end{array}\right]^{T} \in \mathbb{R}^{\bar{n}}, \bar{n}=n+k l
$$$$
\bar{E}=\left[\begin{array}{ccccc}
E & 0 & \cdots & 0 & 0 \\
0 & I_{k} & \cdots & 0 & 0 \\
\vdots & \vdots & \ddots & \vdots & \vdots \\
0 & 0 & \cdots & I_{k} & 0 \\
0 & 0 & \cdots & 0 & I_{k}
\end{array}\right], \bar{A}_{i}=\left[\begin{array}{ccccc}
A_{i} & 0 & \cdots & 0 & F_{i} \\
0 & 0 & \cdots & 0 & 0 \\
\vdots & I_{k} & \ddots & \vdots & \vdots \\
\vdots & \vdots & \ddots & \vdots & \vdots \\
0 & 0 & \cdots & I_{k} & 0
\end{array}\right]
$$$$
\bar{B}_{i}=\left[\begin{array}{c}
B_{i} \\
0 \\
\vdots \\
0
\end{array}\right], \bar{R}_{i}=\left[\begin{array}{cc}
R_{i} & 0 \\
0 & I_{k} \\
\vdots & \vdots \\
0 & 0
\end{array}\right], \bar{\Delta}_{i}=\left[\begin{array}{c}
\Delta x_{i} \\
0 \\
\vdots \\
0
\end{array}\right]
$$

$$
\bar{w}(t)=\left[\begin{array}{c}
w(t) \\
0 \\
\vdots \\
0
\end{array}\right], \bar{d}(t)=\left[\begin{array}{c}
d(t) \\
f^{(t)}(t)
\end{array}\right], \bar{G}=\left[\begin{array}{ll}
G & 0 \ldots 0
\end{array}\right]
$$

and $\bar{C}=\left[\begin{array}{lll}C & 0 \ldots 0\end{array}\right]$.

Consider the following multi-observer for the augmented descriptor multi-models described by (57):

$$
\left\{\begin{array}{l}
\dot{\bar{z}}(t)=\sum_{i=1}^{h} h_{i}(\hat{x}(t))\left[\bar{N}_{i} \bar{z}(t)+\bar{G}_{i} u(t)+\bar{L}_{i} y(t)+\bar{\Delta} \bar{z}_{i}\right] \\
\left.\hat{\bar{x}}(t)=\bar{z}(t)+\bar{T}_{2} y(t)\right)
\end{array}\right.
$$

where $\bar{z}(t) \in \mathbb{R}^{\bar{n}}$ is the state of the multi-observer and $\hat{\bar{x}}(t) \in \mathbb{R}^{\bar{n}}$ is the estimated state of the augmented state vector $\bar{x}(t) . \bar{N}_{i}, \bar{L}_{i}, \bar{G}_{i}$ and $\bar{T}_{2}$ are matrices with appropriate dimensions. If the following conditions hold true $\forall i=1, \ldots, h$ :

$$
\begin{aligned}
& \bar{T}_{1} \bar{A}_{i}=\bar{N}_{i} \bar{T}_{1} \bar{E}+\bar{L}_{i} \bar{C} \\
& \bar{G}_{i}=\bar{T}_{1} \bar{B}_{i} \\
& \bar{\Delta} \bar{z}_{i}=\bar{T}_{1} \bar{\Delta}_{\bar{\Delta}} \bar{x}_{i} \\
& \left(\bar{L}_{i}-\bar{N}_{i} \bar{T}_{2}\right) \bar{G}-\bar{T}_{1} \bar{R}_{i}=0 \\
& \bar{T}_{2} \bar{G}=0 \\
& I_{\bar{n}}=\bar{T}_{1} \bar{E}+\bar{T}_{2} \bar{C}
\end{aligned}
$$


Then, the dynamic of the state estimation error becomes:

$$
\dot{\bar{e}}(t)=\sum_{i=1}^{h} h_{i}(\hat{\xi}(t))\left[\bar{N}_{i} \bar{e}(t)-\bar{T}_{1} \bar{R}_{i} \bar{d}(t)-\bar{T}_{1} \bar{w}(t)\right]
$$

If the above estimation error (65) is stable, the augmented state estimated $\hat{\bar{x}}(t)$ will converge to the real state $\bar{x}(t)$.

Theorem 3: For $\bar{d}(t)$ and $\bar{w}(t)$ with bounded norms, the multi-models description (58) is a robust observer for the augmented descriptor multi-models (57) such that:

$$
\|\bar{e}(t)\|_{2} \leq \eta\|\bar{w}(t)\|_{2}
$$

if there exists a common positive definite matrix $\bar{Q}$ such that:

$$
\left[\begin{array}{ccc}
\bar{\Pi}_{i} & \bar{Q}\left(\bar{T}_{1} \bar{R}_{i}\right) & -\bar{Q} \bar{T}_{1} \\
\left(\bar{T}_{1} \bar{R}_{i}\right)^{T} \bar{Q} & -\frac{1}{\eta_{1}} I_{q+k} & 0 \\
-\bar{T}_{1}^{T} \bar{Q} & 0 & I_{\bar{n}}
\end{array}\right]<0, \forall i=1, \ldots, h
$$

s.t.

$$
\bar{W}_{i} \bar{G}+\bar{Q} \bar{T}_{1} \bar{R}_{i}=0
$$

where $\eta_{1}=\alpha^{-1} \eta^{2}$ and

$$
\bar{\Pi}_{i}=\left(\bar{T}_{1} \bar{A}_{i}\right)^{T} \bar{Q}+\bar{Q}\left(\bar{T}_{1} \bar{A}_{i}\right)+\left(\alpha-\gamma^{2}\right) I_{\bar{n}}+\left(\bar{W}_{i} \bar{C}\right)^{T}+\left(\bar{W}_{i} \bar{C}\right)
$$

Proof: The proof is similar as for Theorem 2, and is thus omitted.

As a result, the estimated state vector includes the components such as the estimated state $\hat{x}(t)$, the estimates $\hat{\zeta}_{i}(t)(i=1,2, \ldots, l-1)$ for the derivatives of the fault, and the fault estimation $\hat{\zeta}_{l}(t)=\hat{f}(t)$. Therefore, the augmented observer (58) is a simultaneous state and fault estimator.

\section{APPLICATION: AN ELECTRICAL CIRCUIT}

The studied process is an electrical circuit [11] shown in Figure 1.

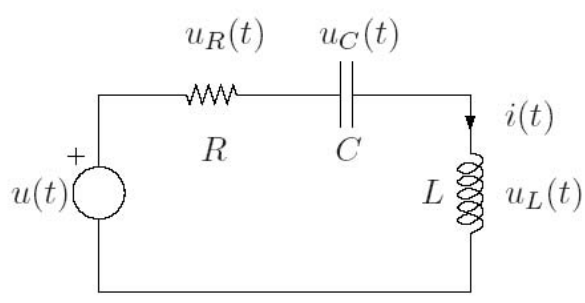

Fig 1: An electrical circuit with an ideal voltage source and three nonlinear components.

This circuit consists of a voltage source, a resistor, a capacitor and an inductor. The voltage source is assumed to be ideal, i.e. having no internal resistance and giving the voltage $u(t)$. The inductor is assumed to have a ferromagnetic core resulting in a saturated magnetic flux $\Phi(t)$ for large currents $i(t)$.

If the notation $x_{1}(t)=u_{C}(t), x_{2}(t)=\phi(t), x_{3}(t)=i(t)$ and $x_{4}(t)=u_{L}(t)$ are used, then the system can be described by the following nonlinear descriptor model:

$$
\left\{\begin{aligned}
\dot{x}_{1}(t) & =\frac{x_{3}(t)}{1+0.01 x_{1}(t)} \\
\dot{x}_{2}(t) & =x_{4}(t) \\
0 & =x_{2}(t)-\arctan \left(x_{3}(t)\right)+d(t) \\
0 & =-x_{1}(t)-x_{3}(t)-x_{3}^{3}(t)-x_{4}(t)+u(t) \\
y(t) & =\left[\begin{array}{llll}
1 & 0 & 1 & 0 \\
0 & 0 & 1 & 0 \\
0 & 1 & 0 & 0
\end{array}\right] x(t)+\left[\begin{array}{c}
0.5 \\
0.8 \\
0
\end{array}\right] d(t)
\end{aligned}\right.
$$

\subsection{Multi-models representation}

The previous nonlinear model can be linearized around four operating points. Then, a descriptor multimodels form can be formulated as follows:

$$
\left\{\begin{array}{c}
E \dot{x}(t)=\sum_{i=1}^{4} h_{i}(\hat{x}(t))\left[A_{i} x(t)+B_{i} u(t)+R_{i} d(t)\right. \\
y(t)=C x(t)+G d(t)
\end{array}\right.
$$

The numerical values of those matrices are given by:

$E=\left[\begin{array}{llll}1 & 0 & 0 & 0 \\ 0 & 1 & 0 & 0 \\ 0 & 0 & 0 & 0 \\ 0 & 0 & 0 & 0\end{array}\right], A_{1}=\left[\begin{array}{cccc}0.0198 & 0 & 1.0215 & 0 \\ 0 & 0 & 0 & 1 \\ 0 & 1 & -0.2169 & 0 \\ -1 & 0 & -11.83 & -1\end{array}\right]$

$A_{2}=\left[\begin{array}{cccc}0.0075 & 0 & 0.9671 & 0 \\ 0 & 0 & 0 & 1 \\ 0 & 1 & -0.6098 & 0 \\ -1 & 0 & -2.92 & -1\end{array}\right], B_{i}=B=\left[\begin{array}{l}0 \\ 0 \\ 0 \\ 1\end{array}\right]$

$A_{3}=\left[\begin{array}{cccc}-0.0073 & 0 & 0.9579 & 0 \\ 0 & 0 & 0 & 1 \\ 0 & 1 & -0.6098 & 0 \\ -1 & 0 & -2.92 & -1\end{array}\right], R_{1}=R_{3}=\left[\begin{array}{l}0 \\ 0 \\ 1 \\ 0\end{array}\right]$

$A_{4}=\left[\begin{array}{cccc}-0.0189 & 0 & 1.0173 & 0 \\ 0 & 0 & 0 & 1 \\ 0 & 1 & -0.2301 & 0 \\ -1 & 0 & -11.0357 & -1\end{array}\right], R_{2}=R_{4}=\left[\begin{array}{l}0 \\ 0 \\ 0.5 \\ 0\end{array}\right]$

$C=\left[\begin{array}{llll}1 & 0 & 1 & 0 \\ 0 & 0 & 1 & 0 \\ 0 & 1 & 0 & 0\end{array}\right], G=\left[\begin{array}{c}0.5 \\ 0.8 \\ 0\end{array}\right], \Delta x_{1}=\left[\begin{array}{c}0.04 \\ 0 \\ 0.67 \\ -17.6\end{array}\right]$

$\Delta x_{2}=\left[\begin{array}{c}-0.02 \\ 0 \\ -0.48 \\ 5.94\end{array}\right], \Delta x_{3}=\left[\begin{array}{c}0.03 \\ 0 \\ 0.27 \\ 1.15\end{array}\right], \Delta x_{4}=\left[\begin{array}{c}-0.03 \\ 0 \\ -0.65 \\ 12.6\end{array}\right]$

Let consider the studied case $\mathbf{B}$ where the decision variable is unmeasurable, the weighting functions depend on 
the estimated state $\hat{x}_{4}(t)$ as follows:

$$
h_{i}\left(\hat{x}_{4}(t)\right)=\frac{\mu_{i}\left(\hat{x}_{4}(t)\right)}{\sum_{i=1}^{4} \mu_{i}\left(\hat{x}_{4}(t)\right)}
$$

where $\mu_{i}\left(\hat{x}_{4}(t)\right)$ are defined by

$$
\begin{aligned}
& \mu_{1}\left(\hat{x}_{4}(t)\right)=\exp \left(-1 / 2\left(\frac{\hat{x}_{4}+2}{2}\right)^{2}\right) \\
& \mu_{2}\left(\hat{x}_{4}(t)\right)=\exp \left(-1 / 2\left(\frac{\hat{x}_{4}-2}{2}\right)^{2}\right) \\
& \mu_{3}\left(\hat{x}_{4}(t)\right)=\exp \left(-1 / 2\left(\frac{\hat{x}_{4}+3}{2}\right)^{2}\right) \\
& \mu_{4}\left(\hat{x}_{4}(t)\right)=\exp \left(-1 / 2\left(\frac{\hat{x}_{4}-3}{2}\right)^{2}\right)
\end{aligned}
$$

For $u(t)=10 \sin (0,2 \pi t)$ and $d(t)$ a gaussian signal of variance 1 and zero average, the characterization of the global behavior of the nonlinear system by the multimodels is obtained after the estimation of the state vector to reconstruct the weighting functions. The outputs of the nonlinear system and the multi-models are shown in the following Figures (2-4).

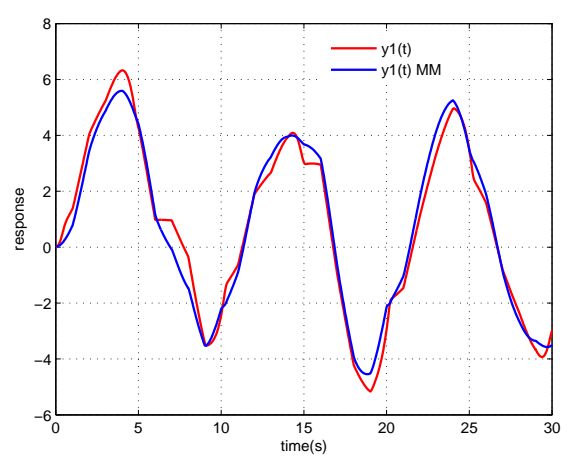

Fig 2: Nonlinear and multi-models outputs

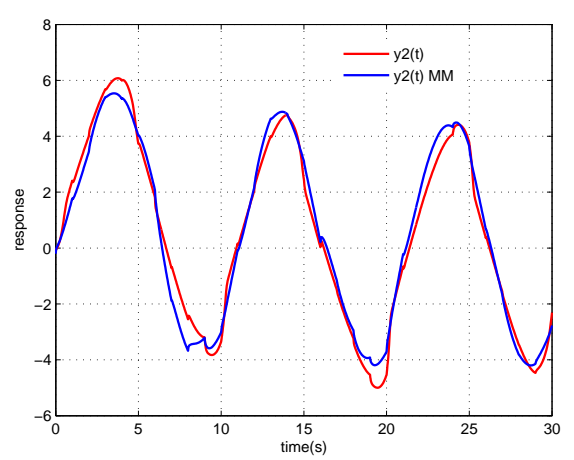

Fig 3: Nonlinear and multi-models outputs

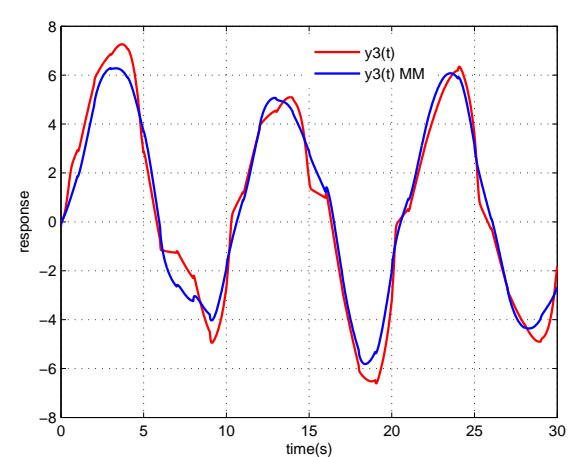

Fig 4: Nonlinear and multi-models outputs

These figures consider the multi-models form with the nonlinear system. They illustrate the superposition of the nonlinear outputs with those coming from the multimodels representation. We can see that the multi-models well approximate the nonlinear dynamic behavior.

\subsection{Fault detection}

In this paragraph, the main task of fault detection is to generate a residual signal which is insensitive to the system disturbances. To detect the fault, the residual has to be sensitive to this fault. According to the study in section (3.), the descriptor multi-models (70) with unknown input and fault can be described as:

$$
\left\{\begin{array}{r}
E \dot{x}(t)=\sum_{i=1}^{4} h_{i}\left(\hat{x}_{4}(t)\right)\left[A_{i} x(t)+B_{i} u(t)+R_{i} d(t)+F_{i} f(t)\right. \\
\left.+\Delta x_{i}+w(t)\right] \\
y(t)=C x(t)+G d(t)
\end{array}\right.
$$

where $F_{i}=F=\left[\begin{array}{cccc}0 & 0 & 0 & 1\end{array}\right]^{T}$ is the fault vector and $w(t)$ is defined as (45). The system is subjected of fault $f(t)$ of the following form:

$$
f(t)=(4-4 \exp (-0,2(t-20))) \varepsilon(t)
$$

where

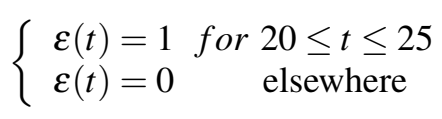

and the unknown input $d(t)$ is a white Gaussian noise with variance 0.01 and zero average.

Second, to design the residual generator (6), it can be shown that conditions (4) and (5) are satisfied. Using (26), one can obtain matrices $T_{1}$ and $T_{2}$ and the LMIs (47) can be efficiently solved under constraints (48) via numerical approach within the LMI toolbox in order to compute the gains matrices. Therefore, these inequalities are fulfilled with:

$$
K_{1}=\left[\begin{array}{ccc}
-0.9402 & 1.0634 & 0 \\
-0.0036 & 0.0022 & -177.4767 \\
1.1739 & -2.0024 & 0 \\
0 & 0 & -0.0072
\end{array}\right]
$$




$$
\begin{aligned}
& K_{2}=\left[\begin{array}{ccc}
-0.9144 & 1.0595 & -0.0002 \\
0.0680 & -0.0425 & -177.4767 \\
1.1050 & -1.9920 & -0.0001 \\
0 & 0 & -0.0072
\end{array}\right] \\
& K_{3}=\left[\begin{array}{ccc}
-0.8985 & 1.0506 & -0.0001 \\
0.0174 & -0.0109 & -177.4767 \\
1.0628 & -1.9681 & 0 \\
0 & 0 & -0.0072
\end{array}\right] \\
& K_{4}=\left[\begin{array}{ccc}
-0.9043 & 1.0385 & 0 \\
-0.0046 & 0.0029 & -177.4767 \\
1.0781 & -1.9359 & 0 \\
0 & 0 & -0.0072
\end{array}\right]
\end{aligned}
$$

The result of the residual signal response is shown in the following figure.

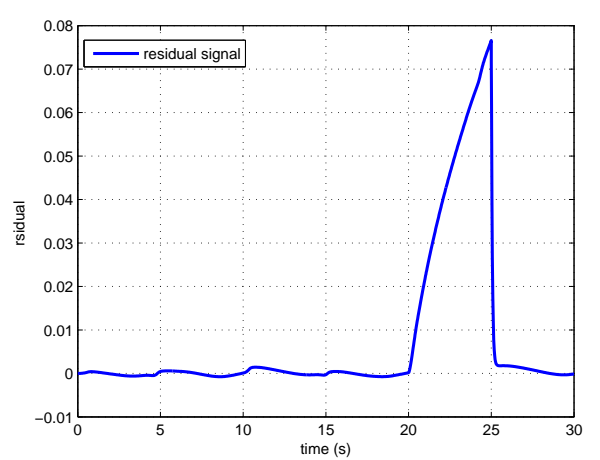

Fig 5: The residual signal $r(t)$

It's clear from the above figure (5) that the residual is almost zero throughout the time simulation run for faultfree residuals despite noisy environment and increase in magnitude considerably, when fault occurs between time $t=20 s$ and $t=25 s$.

\subsection{Fault estimation}

The occurred fault can be approximated by a polynomial function as follows:

$$
f(t)=\left(0.8(t-20)-0.08(t-20)^{2}\right) \varepsilon(t)
$$

Let us construct the augmented descriptor multi-models as defined in (57) with $l=2$ and solving the LMIs (67) under constraints (68), one can obtain:

$$
\bar{K}_{1}=\left[\begin{array}{ccc}
2.1808 & 10.4296 & 0 \\
0 & 0 & -9.5122 \\
-8.0280 & -25.1471 & 0 \\
0 & 0 & -0.2532 \\
19.6191 & 71.1435 & 0 \\
12.6064 & 47.1630 & 0
\end{array}\right]
$$

$$
\begin{aligned}
\bar{K}_{2} & =\left[\begin{array}{ccc}
2.2019 & 10.4594 & 0 \\
0 & 0 & -9.5122 \\
-8.0821 & -25.2203 & 0 \\
0 & 0 & -0.2532 \\
19.6683 & 71.0525 & 0 \\
12.6376 & 47.1054 & 0
\end{array}\right] \\
\bar{K}_{3} & =\left[\begin{array}{ccc}
2.2065 & 10.4582 & 0 \\
0 & 0 & -9.5122 \\
-8.0946 & -25.2202 & 0 \\
0 & 0 & -0.2532 \\
19.6051 & 71.0872 & 0 \\
12.5975 & 47.1274 & 0 \\
2.1852 & 10.4133 & 0 \\
0 & 0 & -9.5122 \\
-8.0417 & -25.1155 & 0 \\
0 & 0 & -0.2532 \\
19.4099 & 71.2857 & 0 \\
12.4738 & 47.2531 & 0
\end{array}\right]
\end{aligned}
$$

These gains matrices are used to determine the remaining matrices of the augmented multi-observer. The estimated fault is then, given by the following figure (6).

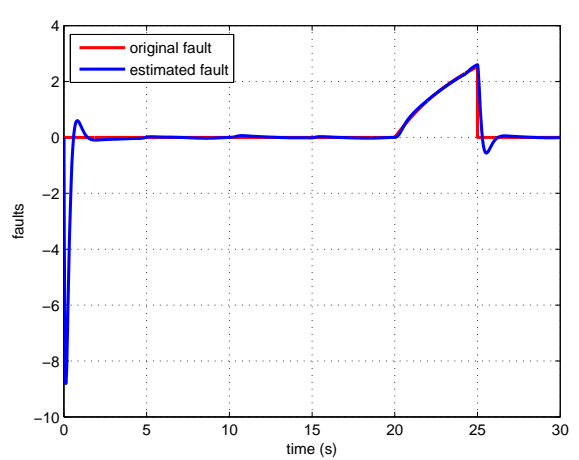

Fig 6: The original fault and its estimated

From the above simulation results, it has been shown that the fault reconstruction algorithm is effective in estimating the fault behavior, for a descriptor system subject to a large class of fault signals such as time-invariant and time-varying signals.

\section{CONCLUSION}

For nonlinear descriptor systems that can be represented by a set of sub-models, a multi-models residual generation is designed. This concept is used for fault detection and estimation problems for descriptor multimodels. To estimate the fault, an augmented residual generator is designed for an augmented descriptor multi-models. Sufficient conditions for the existence of residual generator have been established and the analysis of the stability have been also designed for two cases. One case is dedicated when the decision 
variable of the weighting function do not depend on the unmeasured state variables. The other case is dedicated when the decision variable depends on the unmeasured state variables. The method has been successfully applied to experimental data coming from an electrical circuit with an ideal voltage source and three nonlinear components.

\section{REFERENCES}

[1] A. G. Wu and G.-R. Duan, "Design of PD Observers in Descriptor Linear Systems", International Journal of Control, Automation, and Systems, vol. 5, no. 1, pp. 93-98, February 2007.

[2] A. M. Nagy Kiss, B. Marx, G. Mourot, G. Schutz and J. Ragot "State estimation of two-time scale multiple models. Application to wastewater treatment plant", Control Engineering Practice, vol. 19, pp 1354-1362, 2011.

[3] A. T. Vemuri, M. M. Polycarpou and A. R. Ciric "Fault Diagnosis of Differential-Algebraic Systems", IEEE Transactions on systems, man, and cybernetics, part a: systems and humans, vol. 31, no. 2, pp 143-152, march 2001.

[4] B. Marx, D. Koenig and J. Ragot, "Design of observers for Takagi-Sugeno descriptor systems with unknown inputs and application to fault diagnosis", IET Control Theory Appl., Vol. 1, No. 5, pp. 14871495, September 2007

[5] D. Ichalal, B. Marx, J. Ragot and D. Maquin, "Fault diagnosis for Takagi-Sugeno nonlinear systems", the 7th IFAC Symposium on Fault Detection, Supervision and Safety of Technical Processes, Bacelona, Spain, pp 504- 509, June 30 July 3, 2009.

[6] D. Koenig, "Observer Design for Unknown Input Nonlinear Descriptor Systems via Convex Optimization", IEEE Transactions on Automatic control, vol. 51, no. 6, pp 1047- 1052, June 2006

[7] D. Theilliol, H. Noura and J. C. Ponsart, "Fault diagnosis and accommodation of three-tank system based on analytical redundancy", ISA Transactions, vol. 41, pp 365-382, 2002.

[8] H. Hamdi, M. Rodrigues, C. Mechmeche, D. Theilliol and N. BenHadj Braiek, "Fault detection and isolation in linear parameter-varying descriptor systems via proportional integral observer", International journal of adaptive control and signal processing, Vol. 26, pp 224-240, 2012.

[9] H. Hamdi, M. Rodrigues, C. Mechemeche, D. Theilliol and N. Benhadj Braiek, "State Estimation for Polytopic LPV Descriptor Systems: Application to Fault Diagnosis" the 7th IFAC Symposium on Fault Detection, Supervision and Safety of Technical Processes, Barcelona, Spain, June 30 - July 3 , 2009.

[10] J. L. Chen, "Robust ESPR Analysis and Control for Uncertain Continuous-time Descriptor Systems ", International Journal of Control, Automation, and Systems, vol. 8, no. 1, pp.8-15, 2010.

[11] J. Sjöberg, "Some Results On Optimal Control for Nonlinear Descriptor Systems", Link?ping, Sweden Studies in Science and Technology, Thesis No. 1227, 2006.

[12] M. Boutayeb and M. Darouach, "Observers design for non linear descriptor systems", Proceedings of the 34th Conference on Decision and Control, New Orleans, pp 2369-2374, LA - December 1995

[13] M. Darouach, M. Boutayeb, "Design of Observers for Descriptor Systems", IEEE transactions on Automatic Control, vol. 40. no. 7, pp 1323-1327, 1995.

[14] M. Rodrigues, D. Theilliol, M. Adam-Medina and D. Sauter, "A Fault Detection and Isolation Scheme for Industrial Systems based on Multiple Operating Models", Control Engineering Practice, Vol 16, pp 225-239, 2008.

[15] M. Rodrigues, D. Theilliol and D. Sauter, "Design of a Robust Polytopic Unknown Input Observer for FDI: Application to Nonlinear Systems described by a Multi-Models Representation", 44th IEEE Conference on Decision and Control and European Control Conference ECC, Sevilla, Spain, pp 6268-6273, December 2005.

[16] R. Orjuela and B. Marx and D. Maquin and J. Ragot, "State estimation for nonlinear systems using decoupled multiple model", International Journal of Modelling, Identification and Control, vol. 4, no. 1, pp 59-67, 2008.

[17] S. Mattson, H. Elmqvist, and M. Otter. "Physical system modeling with modelica", Control Engineering Practice, vol. 6, pp 501-510, 1998.

[18] T. Taniguchi, K. Tanaka, and H. O. Wang, "Fuzzy Descriptor Systems and Nonlinear Model Following Control", IEEE Transactions on Fuzzy Systems, vol. 8, no. 4, pp 442-452, August 2000.

[19] Y. Wang, Z. Q. Sun and F. C. Sun, "Robust Fuzzy Control of a Class of Nonlinear Descriptor Systems with Time-Varying Delay", International Journal of Control, Automation and Systems, Vol. 2, No. 1, pp 76-82, March 2004.

[20] Z. Gao and S. X. Ding, "Fault Reconstruction for Lipschitz Nonlinear Descriptor Systems via Linear Matrix Inequality Approach" Circuits Syst Signal Process, vol. 27, pp 295-308, 2008.

[21] Z. Gao and S. X. Ding, "Actuator fault robust estimation and fault-tolerant control for a class of nonlinear descriptor systems" Automatica, vol. 43, pp 912-920, 2007. 